\title{
New Method to Characterize Subgame Perfect Nash Equilibria in Differential Games
}

\author{
J. P. Rincón-Zapatero, ${ }^{1}$ J. Martínez, ${ }^{2}$ and G. Martin-Herrán ${ }^{3}$
}

Communicated by G. Leitmann

\begin{abstract}
In this paper, we present a method for computing Nash equilibria in feedback strategies. This method gives necessary and sufficient conditions to characterize subgame perfect equilibria by means of a system of quasilinear partial differential equations. This characterization allows one to know explicitly the solution of the game in some cases. In other cases, this approach makes a qualitative study easier. We apply this method to nonrenewable resource games.
\end{abstract}

Key Words. Differential games, subgame perfect Nash equilibria, quasilinear partial differential equations, characteristic systems.

\section{Introduction}

Since the creation of game theory by Von Neumann in 1928 , remarkable efforts have been made in the development of this discipline. The theory of differential games provides an adequate framework for the treatment of problems appearing in fields as different as economics, engineering, or biology (Refs. $1-3$ ).

There are a great number of applications that use open-loop Nash equilibria in the literature on nonzero-sum differential games. Due to its complexity, the analysis of equilibria based on feedback rules is less usual, so Nash equilibrium is not subgame perfect.

In this paper, we propose a new approach for the study and determination of Nash equilibrium in feedback strategies. This approach is based on

\footnotetext{
${ }^{1}$ Assistant Professor, Departamento de Economía Aplicada (Matemáticas), Facultad de Ciencias Económicas, Universidad de Valladolid, Valladolid, Spain.

${ }^{2}$ Assistant Professor, Departamento de Economía Aplicada (Matemáticas), Facultad de Ciencias Económicas, Universidad de Valladolid, Valladolid, Spain.

${ }^{3}$ Assistant Professor, Departamento de Economía Aplicada (Matemáticas), Facultad de Ciencias Económicas, Universidad de Valladolid, Valladolid, Spain.
} 
the papers of Bourdache-Siguerdidjane and Fliess (Refs. 4-5) for nonlinear optimal control problems. It consists of characterizing a Nash equilibrium by means of a system of quasilinear partial differential equations. This system is deduced from the application of the maximum principle of Pontryagin by means of the elimination of the costate vectors that appear in the Hamiltonians associated with the players.

Section 2 states a differential game in general form. In Section 3, we apply the necessary condition of the maximum principle of Pontryagin, yielding a new optimality condition for the Nash equilibrium. In Section 4, we prove that this necessary condition is also sufficient, taking into account appropriate hypotheses on the players' Hamiltonians. These hypotheses are not as strict as the concavity sufficient conditions that are usually used in this kind of problems. The theorem proved in this section also shows that the Nash equilibrium is subgame perfect. In Section 5, we apply the results to nonrenewable resource differential games. After giving a condition that guarantees the existence and uniqueness of a Nash equilibrium, a qualitative study is performed. We include a comparative analysis of solutions obtained when considering variations in the model.

\section{Description of the Game}

We consider an $\mathrm{N}$-person noncooperative differential game over a fixed bounded time interval, namely,

$$
\begin{array}{ll}
\max _{u^{\prime}} & \left\{J^{i}\left(t_{0}, x_{0}, u^{1}, \ldots, u^{N}\right)=\int_{t_{0}}^{t_{f}} L^{i}\left(t, x, u^{1}, \ldots, u^{N}\right) d t+S^{i}\left(t_{f}, x\left(t_{f}\right)\right)\right\}, \\
\text { s.t. } \quad & \dot{x}=f\left(t, x, u^{1}, \ldots, u^{N}\right), \\
& x\left(t_{0}\right)=x_{0}, \\
& u^{i}(t) \in U^{i}, \quad \forall t \in\left[t_{0}, t_{f}\right], \quad U^{i} \text { an open subset of } \mathbb{R}^{n} .
\end{array}
$$

Here, the functions $L^{i}, f, S^{i}$ are assumed to be twice continuously differentiable. The vectors $x=\left(x^{1}, \ldots, x^{n}\right)^{T} \in \mathbb{R}^{n}$ and $u^{i}=\left(u_{1}^{i}, \ldots, u_{n}^{i}\right)^{T}$ represent the state and control variables for the $i$ th player, respectively, where ${ }^{T}$ denotes the transposition sign, $u^{i}(t)=\phi^{i}(t, x(t))$, the function $\phi^{i}:\left[t_{0}, t_{f}\right] \times \mathbb{R}^{n} \rightarrow U^{i}$ is supposed to be differentiable and $u^{i}$ denotes the set of functions $\phi^{i}$.

The system

$$
\begin{aligned}
& \dot{x}=f\left(t, x, \phi^{l}(t, x), \ldots, \phi^{N}(t, x)\right), \\
& x\left(t_{0}\right)=x_{0},
\end{aligned}
$$


is the closed-loop system associated with the $N$-tuple of feedback strategies $\phi=\left(\phi^{1}, \ldots, \phi^{N}\right)^{T}$.

An $N$-tuple of strategies $\hat{\phi}$ is called a subgame perfect equilibrium of the game if, for all $i=1, \ldots, N$,

$$
\begin{aligned}
& J^{i}\left(t, x,\left(\phi^{i} \mid \hat{\phi}_{-i}\right)\right) \\
& \leq J^{i}(t, x, \hat{\phi}), \quad \text { for all } \phi^{i} \text { and for all } t, x \text { admissible, }
\end{aligned}
$$

where $\hat{\phi}_{-i}$ denotes $\left(\hat{\phi}^{1}, \ldots, \hat{\phi}^{i-1}, \hat{\phi}^{i+1}, \ldots, \hat{\phi}^{N}\right)$ and

$$
\left(\phi^{i} \mid \hat{\phi}_{-i}\right)=\left(\hat{\phi}^{1}, \ldots, \hat{\phi}^{i-1}, \phi^{i}, \hat{\phi}^{i+1}, \ldots, \hat{\phi}^{N}\right) .
$$

\section{Necessary Conditions for Optimality}

In this section, as a consequence of applying the maximum principle, we derive a system of quasilinear first-order partial differential equations that must be satisfied for smooth Nash equilibrium. In the following, we use the subscript notation for partial differentiation, with the common convention that the partial derivative of a scalar function with respect to a vector and the partial derivative of a vector function with respect to a scalar are defined as column vectors. Also, the partial derivative of a vector function with respect to another vector is defined as a matrix, e.g.,

$$
h_{z}=\partial h / \partial z=\left[\partial h^{i} / \partial z^{j}\right]_{n \times m},
$$

where $h$ and $z$ are $n \times 1$ and $m \times 1$ vectors, respectively.

We define the Hamiltonian functions

$$
H^{i}\left(t, x, u, \mu^{i}\right)=L^{i}(t, x, u)+f^{T}(t, x, u) \mu^{i},
$$

where $\mu^{i}=\left(\mu_{1}^{i}, \ldots, \mu_{n}^{i}\right)^{T}$ is the vector of costate variables associated to the $i$ th player. By the maximum principle, if $\hat{\phi}$ is a Nash equilibrium of (1)-(3), differentiable functions $\lambda^{i}$ exist, satisfying the following two-point boundaryvalue problem:

$$
\begin{aligned}
& \dot{\lambda}^{i}=-\hat{H}_{x}^{i}-\hat{\phi}_{x}^{r} \hat{H}_{u}^{i}, \\
& \dot{x}=\hat{H}_{\mu^{i}}^{i}, \\
& \lambda\left(t_{f}\right)=S_{x}^{i}\left(t_{f}, x\left(t_{f}\right)\right), \\
& x\left(t_{0}\right)=x_{0},
\end{aligned}
$$

where $\hat{H}_{\{\cdot\}}^{i}$ means $H_{\{\cdot\}}^{i}$ evaluated at $\left(t, x, \hat{\phi}, \lambda^{i}\right)$. 
Moreover, $\hat{\phi}^{i}$ maximizes the Hamiltonian of the $i$ th player, so that the following inequality holds:

$$
H^{i}\left(t, x,\left(\phi^{i} \mid \hat{\phi}_{-i}\right), \lambda^{i}\right) \leq H^{i}\left(t, x, \hat{\phi}, \lambda^{i}\right), \quad \forall \phi^{i}, i=1, \ldots, N .
$$

Due to the fact that there are no constraints over the admissible controls, this last condition implies

$$
\begin{aligned}
\hat{H}_{u^{i}}^{i} & =H_{u^{i}}^{i}\left(t, x, \hat{\phi}, \lambda^{i}\right) \\
& =L_{u^{i}}^{i}(t, x, \hat{\phi})+f_{u^{\prime}}^{T_{i}}(t, x, \hat{\phi}) \lambda^{i}=0, \quad \forall t \in\left[0, t_{f}\right], i=1, \ldots, N .
\end{aligned}
$$

The system (8) is linear in the costate variables $\lambda_{l}^{i}, \ldots, \lambda_{n}^{i}$, for each $i$. Then, (8) admits a unique solution in these variables if and only if the determinant of $f_{u^{i}}$ is nonzero. In this case, $\lambda_{i}$ can be regarded as a function of $(t, x)$, so we can write

$$
\lambda^{i}=-\left(f_{u^{i}}^{T}\right)^{-1} L_{u^{i}}^{i}
$$

Since (8) holds for all $t \in\left[t_{0}, t_{f}\right]$, we have

$$
(d / d t) \hat{H}_{u^{i}}^{i}=0, \quad \forall i=1, \ldots, N .
$$

Substituting in (10) the expressions for $\dot{x}$ and $\dot{\lambda}^{i}$ given by Eqs. (4), (5) we obtain, for each $i=1, \ldots, N$,

$$
\begin{aligned}
0 & =(d / d t) H_{u^{\prime}}^{i}\left(t, x, \hat{\phi}(t, x), \lambda^{i}\right) \\
& =\hat{H}_{u^{\prime} t}^{i}+\hat{H}_{u^{\prime} x}^{i} \dot{x}+\hat{H}_{u^{i} u}^{i}\left(\hat{\phi}_{t}+\hat{\phi}_{x} \dot{x}\right)+\hat{H}_{u^{i} \mu^{i}}^{i} \dot{\lambda}^{i} \\
& =\hat{H}_{u^{\prime} t}^{i^{i}}+\hat{H}_{u^{i} x}^{i^{i}} f+\hat{H}_{u^{\prime} u}^{i}\left(\hat{\phi}_{t}+\hat{\phi}_{x} f\right)+\hat{H}_{u^{\prime} \mu^{\prime}}^{i^{i}}\left(-\hat{H}_{x}^{i}-\hat{\phi}_{x}^{T} \hat{H}_{u}^{i}\right) .
\end{aligned}
$$

By replacing (9) in the expression above, we get

$$
\begin{aligned}
& {\left[L_{u^{\prime} u}^{i}-f_{u^{\prime} u}^{T}\left(I_{n N \times n N} \otimes\left(f_{u^{\prime}}^{-T} L_{u^{i}}^{i}\right)\right)\right]\left[\hat{\phi}_{\imath}+\hat{\phi}_{x} f\right]} \\
& \quad-f_{u^{i}}^{T} \hat{\phi}_{x}^{T}\left[L_{u}^{i}-f_{u}^{T} f_{u^{i}}^{-T} L_{u^{i}}^{i}\right] \\
& =-L_{u^{i} \iota}^{i}+f_{u^{\prime} t}^{T} f_{u^{i}}^{-T} L_{u^{\prime}}^{i}-L_{u^{\prime} x}^{i} f+f_{u^{\prime}}^{T} L_{x}^{i} \\
& \quad+f_{u x}^{T}\left(I_{n \times n} \otimes\left(f_{u^{\prime}}^{-T} L_{u^{i}}^{i}\right)\right) f-f_{u^{i}}^{T} f_{x}^{T} f_{u^{u^{\prime}}}^{-T} L_{u^{i}}^{i},
\end{aligned}
$$

where $I_{n N \times n N}$ is the $n N$-dimensional identity matrix; the symbol $\otimes$ denotes the Kronecker product, defined as follows for the matrices $A=\left(a_{i j}\right)_{p \times q}$ and $B=\left(b_{i j}\right)_{r \times s}$ :

$$
A \otimes B=a_{i j} B
$$

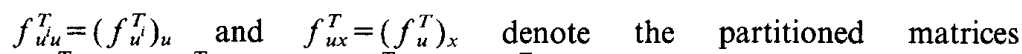
$\left(f_{u}^{T_{i} u \mid}|\cdots| f_{u^{\prime} u_{N}}^{T_{N}}\right)$ and $\left(f_{u^{i} x_{1}}^{T_{1}} \cdots \mid f_{u^{\prime} x_{n}}^{T_{i}}\right)$, respectively. Hence, we have obtained a system of $n N$ quasilinear partial differential equations that must be verified for a sufficiently smooth feedback Nash equilibrium. 
From the boundary conditions (6) and (8), we can provide a complete set of final conditions for the system (11) given by

$$
L_{u^{\prime}}^{i}\left(t_{f}, x, \hat{\phi}\right)+f_{u^{\prime}}^{T^{\prime}}\left(t_{f}, x, \hat{\phi}\right) S_{x}^{i}\left(t_{f}, x\right)=0 .
$$

Remark 3.1. It can be observed that, when $N=1$ (e.g., the problem is an optimal control problem), (11) is the expression required in Ref. 5. In this case, (11) is a system of quasilinear partial differential equations with the same principal part, using the terminology of Courant and Hilbert (Ref. 6). This property permits characterizing the solution of (11), (12) by means of a system of ordinary differential equations, called the characteristic system, which is developed by Bourdache and Fliess (Ref. 5). Unfortunately, this is not possible when $N>1$, because in that case the equations are coupled due to the effects of the cross-influence terms in (4).

Remark 3.2. We point out the fact that, in the scalar case $(n=1)$, the matricial form (11) is reduced to

$$
A \hat{\phi}_{t}+B \hat{\phi}_{x}=C,
$$

with matrices

$$
A=\left(a_{i j}\right)_{N \times N}, \quad B=\left(b_{i j}\right)_{N \times N}, \quad C=\left(c_{i}\right)_{N \times l},
$$

whose components are given by

$$
\begin{aligned}
& a_{i j}=L_{u^{\prime} u^{\prime}}^{i}-f_{u^{\prime} u^{j}} f_{u^{\prime}}^{-1} L_{u^{\prime}}^{i}, \\
& b_{i j}=\left(L_{u^{\prime} u^{j}}^{i}-f_{u^{\prime} u^{\prime}} f_{u^{\prime}}^{-1} L_{u^{\prime}}^{i}\right) f-f_{u^{\prime}} L_{u^{j}}^{i}+f_{u^{\prime}} L_{u^{\prime}}^{i}, \\
& c_{i}=-L_{u^{\prime} t}^{i}+f_{u^{\prime}} f_{u^{\prime}}^{-1} L_{u^{\prime}}^{i}-L_{u^{\prime} x} f+f_{u^{\prime}} L_{x}^{i}+f_{u^{\prime}}^{-1} L_{u^{\prime}}^{i} f_{u^{\prime} x} f-L_{u^{\prime}}^{i} f_{x} .
\end{aligned}
$$

From now on we concentrate on the scalar case $n=1$ for the sake of notational simplicity. However, all the results that we present remain valid in the general case.

\section{Sufficient Conditions for Optimality}

The system (13) was obtained by applying a set of necessary conditions for optimality. We can wonder if a classical solution of that system becomes a Nash equilibrium of the differential game. The aim of the following results 
is to show that, under suitable hypotheses about the Hamiltonian functions, that is so. First, let us define the value function of the $i$ th player as

$$
\begin{gathered}
V^{i}(t, x)=\max _{\phi^{i}}\left\{\int_{t}^{t_{f}} L^{i}\left(s, y,\left(\phi^{i} \mid \hat{\phi}_{-i}\right)\right) d s+S^{i}\left(t_{f}, x\left(t_{f}\right)\right) \mid\right. \\
\dot{y}=f\left(s, y,\left(\phi^{i} \mid \hat{\phi}_{-i}\right)\right), s \in\left(t, t_{f}\right) ; y(t)=x, \\
\left.\phi^{i}(s, y(s)) \in \mathscr{U}^{i}, \forall s \in\left(t, t_{f}\right)\right\} .
\end{gathered}
$$

Theorem 4.1. Let $\hat{\phi}$ be a global $\mathscr{C}^{1}$ solution of (13), with final condition (12), satisfying $f_{u^{i}}(t, x, \hat{\phi}) \neq 0$ and

$$
\begin{array}{r}
H^{i}\left(t, x,\left(\phi^{i} \mid \hat{\phi}_{-i}\right), \Gamma^{i}(t, x)\right) \leq H^{i}\left(t, x, \hat{\phi}, \Gamma^{i}(t, x)\right), \\
\forall t \in\left[t_{0}, t_{f}\right], x \in \mathbb{R}, i=1, \ldots, N,
\end{array}
$$

where

$$
\Gamma^{i}(t, x)=-f_{u^{i}}^{-1}(t, x, \hat{\phi}) L_{u^{i}}^{i}(t, x, \hat{\phi}) .
$$

Then, $\Gamma^{i}(t, x)=V_{x}^{i}(t, x)$ and $\hat{\phi}$ is a subgame perfect Nash equilibrium.

Proof. The essential observation is that $\Gamma^{i}(s, y(s))$ is the costate variable of the $i$ th player in the problem

$$
\begin{aligned}
\max _{\phi^{i}} & \int_{t}^{t_{f}} L^{i}\left(s, y,\left(\phi^{i} \mid \hat{\phi}_{-i}\right)\right) d s+S^{i}\left(t_{f}, y\left(t_{f}\right)\right), \\
\text { s.t. } & \dot{y}=f\left(s, y,\left(\phi^{i} \mid \hat{\phi}_{-i}\right)\right), \quad s \in\left(t, t_{f}\right), \\
& y(t)=x, \quad \phi^{i}(s, y(s)) \in \mathscr{U}^{i}, \quad \forall s \in\left(t, t_{f}\right) .
\end{aligned}
$$

Proving that $\Gamma^{i}(s, y(s))$ verifies (4) is straightforward:

$$
\dot{\Gamma}^{i}(s, y(s))=-\hat{H}_{y}^{i}-\hat{\phi}_{y}^{T} \hat{H}_{u}^{i},
$$

taking (13) into account. It turns out that $y, \hat{\phi}, \Gamma^{i}$ satisfy the necessary conditions for optimality, in view of hypothesis (14).

We can readily adapt the proof of the main theorem in Willemstein (Ref. 7) to our framework to obtain the equality

$$
\Gamma^{i}(t, x)=(\partial / \partial x) J^{i}(t, x, \hat{\phi}) .
$$


We have

$$
\begin{aligned}
& (\partial / \partial x) J^{i}(t, x, \hat{\phi}) \\
& =\int_{t}^{t_{f}}(\partial / \partial x) L^{i}(s, y, \hat{\phi}(s, y)) d s+(\partial / \partial x) S^{i}\left(t_{f}, y\left(t_{f}\right)\right) \\
& =\int_{t}^{t_{f}}\left\{L_{y}^{i}(s, y, \hat{\phi}(s, y))(\partial y / \partial x)+\hat{\phi}_{x}^{T}(s, y) L_{u}^{i}(s, y, \hat{\phi}(s, y))\right\} d s \\
& \quad+(\partial / \partial x) S^{i}\left(t_{f}, y\left(t_{f}\right)\right) \\
& =\int_{t}^{t_{f}}\left\{\left[-\dot{\Gamma}^{i}(s, y)-f_{y}(s, y, \hat{\phi}(s, y)) \Gamma^{i}(s, y)\right.\right. \\
& \left.\quad-\quad \hat{\phi}_{y}^{T}(s, y) \hat{H}_{u}^{i}\left(s, y, \hat{\phi}(s, y), \Gamma^{i}(s, y)\right)\right] \\
& \left.\quad \times(\partial y / \partial x)+\hat{\phi}_{x}^{T}(s, y) L_{u}^{i}(s, y, \hat{\phi}(s, y))\right\} d s \\
& =\int_{t}^{t_{f}}\left\{-(\partial y / \partial x) \dot{\Gamma}^{i}(s, y)-(\partial / \partial x) f(s, y, \hat{\phi}(s, y)) \Gamma^{i}(s, y)\right\} d s \\
& \quad+(\partial / \partial x) S^{i}\left(t_{f}, y\left(t_{f}\right)\right) \\
& =-\left.(\partial y / \partial x) \Gamma^{i}(s, y)\right|_{f} ^{t_{f}} \\
& \quad+\int_{t}^{t_{f}}((d / d s)(\partial y / \partial x)-(\partial / \partial x) f(s, y, \hat{\phi}(t, y))) \Gamma^{i}(s, y) d s \\
& \quad+(\partial / \partial x) S^{i}\left(t_{f}, y\left(t_{f}\right)\right) .
\end{aligned}
$$

Now, the derivative with respect to the initial condition satisfies the following equation:

$(d / d t)(\partial y / \partial x)=(\partial / \partial y) f(s, y, \hat{\phi}(t, y))(\partial y / \partial x)$,

and finally we arrive at the desired conclusion,

$$
\begin{aligned}
& (\partial / \partial x) J^{i}(t, x, \hat{\phi}) \\
& =-\left.(\partial y / \partial x) \Gamma^{i}(s, y)\right|_{f} ^{t_{f}}+(\partial / \partial x) S^{i}\left(t_{f}, y\left(t_{f}\right)\right) \\
& =-(\partial y / \partial x)\left(t_{f}\right) \Gamma^{i}\left(t_{f}, y\left(t_{f}\right)\right)+\Gamma^{i}(t, y(t))+(\partial y / \partial x)\left(t_{f}\right) S_{y}^{i}\left(t_{f}, y\left(t_{f}\right)\right) \\
& =\Gamma^{i}(t, x) .
\end{aligned}
$$


In view of hypothesis (14), for any arbitrary strategy $\phi^{i}$, we have

$$
\begin{aligned}
& H^{i}\left(s, z(s),\left(\phi^{i} \mid \hat{\phi}_{-i}\right), J_{x}^{i}(s, z(s), \hat{\phi})\right)+J_{s}^{i}(s, z(s), \hat{\phi}) \\
& \leq H^{i}\left(s, z(s), \hat{\phi}, J_{x}^{i}(s, z(s), \hat{\phi})\right)+J_{s}^{i}(s, z(s), \hat{\phi}),
\end{aligned}
$$

where $z$ is the solution of

$$
\dot{x}=f\left(s, x,\left(\phi^{i} \mid \hat{\phi}_{-i}\right)\right), \quad x(t)=x, \quad t \in\left[t_{0}, t_{f}\right) .
$$

Then, we can write

$$
\begin{aligned}
& (d / d s) J^{i}(s, z(s), \hat{\phi})+L^{i}\left(s, z(s),\left(\phi^{i} \mid \hat{\phi}_{-i}\right)\right) \\
& \leq(d / d s) J^{i}(s, z(s), \hat{\phi})+L^{i}(s, z(s), \hat{\phi})=0 .
\end{aligned}
$$

Integrating the above expression between $t$ and $t_{f}$, we obtain the following inequality:

$$
S^{i}\left(t_{f}, z\left(t_{f}\right)\right)-J^{i}(t, x, \hat{\phi})+\int_{t}^{t_{f}} L^{i}\left(s, z,\left(\phi^{i} \mid \hat{\phi}_{-i}\right)\right) d s \leq 0,
$$

and thus,

$$
J^{i}\left(t, x,\left(\phi^{i} \mid \hat{\phi}_{-i}\right)\right) \leq J^{i}(t, x, \hat{\phi}),
$$

so that

$$
J^{i}(t, x, \hat{\phi})=V^{i}(t, x), \quad \Gamma^{i}(t, x)=V_{x}^{i}(t, x),
$$

and $\hat{\phi}$ is a subgame perfect Nash equilibrium.

Let us note that the concavity of each maximized Hamiltonian with respect to the state variable,

$$
\max _{u^{i}} H^{i}\left(t, x,\left(u^{i} \mid \hat{\phi}_{-i}\right), \mu^{i}\right), \quad \text { for all } t, \mu^{i}, i=1, \ldots, N,
$$

is not required, as is common when the Arrow sufficiency conditions are used.

Remark 4.1. If the function $u^{i} \mapsto H^{i}$ is concave for all $t, x, \mu^{i}, i=$ $1, \ldots, N$, then a global classical solution $\hat{\phi}$ of (13) with final conditions (12) verifies the hypothesis of Theorem 4.1, because

$$
H_{u^{i}}^{i}\left(t, x, \hat{\phi}, \Gamma^{i}\right)=0,
$$

by construction. If the function $u^{i} \mapsto H^{i}$ is strictly concave, then it turns out that only a unique classical solution of (13), (12) can exist and therefore that is a unique Nash equilibrium. 
Remark 4.2. In fact, a global classical solution of (13), (12) verifying hypothesis (14) of the above theorem has the property of strong time consistency or subgame perfection (Ref. 8). The value functions $V^{i}, i=1, \ldots, N$, defined above satisfy the Hamilton-Jacobi-Bellman equations,

$$
\begin{aligned}
& V_{t}^{i}(t, x)=-\max _{\phi^{i}}\left\{L^{i}\left(t, x,\left(\phi^{i} \mid \hat{\phi}_{-i}\right)\right)+V_{x}^{i}(t, x) f\left(t, x,\left(\phi^{i} \mid \hat{\phi}_{-i}\right)\right)\right\}, \\
& V^{i}\left(t_{f}, x\right)=S^{i}\left(t_{f}, x\right) .
\end{aligned}
$$

Remark 4.3. The system (13) can also be used to characterize openloop Nash equilibria. In this case, we consider solutions to the ordinary differential system given by

$$
\begin{aligned}
& (d / d t) \hat{\phi}=A^{-1} C, \\
& (d / d t) x=f,
\end{aligned}
$$

with the final condition determined by the equality

$$
L_{u^{\prime}}{ }^{\prime}\left(t_{f}, x\left(t_{f}\right), \hat{\phi}\left(t_{f}\right)\right)+f\left(t_{f}, x\left(t_{f}\right), \hat{\phi}\left(t_{f}\right)\right)(\partial / \partial x) S^{i}\left(t_{f}, x\left(t_{f}\right)\right)=0 .
$$

Let us observe that, if $(\partial / \partial x) A^{-1} C=0$ and the final condition does not depend on $x$, it is possible that a solution of (13) independent of the state variable exists. If this solution is a Nash equilibrium, it is called degenerate in the literature. Sufficient conditions that assure this property are given in Ref. 9.

Remark 4.4. In the case of an infinite horizon, $t_{f}=\infty$, if

$$
\int_{t_{0}}^{\infty} L^{i}\left(t, x, u^{1}, \ldots, u^{N}\right) d t
$$

converges uniformly for all admissible controls $u^{1}, \ldots, u^{N}$, and if $\hat{\phi}$ is a solution to (13) with $\lim _{t \rightarrow \infty}(\partial y / \partial x)\left(t_{f}\right) \Gamma^{i}(t, x)=0$, then $\hat{\phi}$ is a subgame perfect Nash equilibrium.

When the game problem is autonomous, we can consider solutions to (13) independent of $t$, so that

$$
B(d / d x) \hat{\phi}=C
$$

\section{Nonrenewable Resource Games}

In this section, we analyze existence and uniqueness of subgame perfect Nash equilibrium in finite-horizon nonrenewable resource games. This kind 
of games, but of unbounded horizon, have been widely studied in the literature; see, for example, Ref. 10.

Suppose that a nonrenewable common property resource is exploited by $N$ agents, with $N$ greater than one, in a noncooperative fashion. The evolution of the resource obeys the differential equation

$$
\dot{x}=-\sum_{i=1}^{N} u^{i}, \quad x(0)=x_{0}>0,
$$

where $x_{0}$ is the initial resource endowment. The $i$ th player depletes the resource at a rate $u^{i} \geq 0$ and its payoff is given by

$$
\begin{aligned}
J_{i}\left(0, x_{0}, u\right) & =\int_{0}^{t_{f}} \exp \left(-r_{i} t\right) L_{i}\left(u^{i}\right) d t \\
& +\exp \left(-r_{i} t_{f}\right) S_{i}\left(x\left(t_{f}\right)\right), \quad 0<t_{f}<\infty .
\end{aligned}
$$

We consider $u^{i}(t)=\phi^{i}(t, x(t))$, with $\phi^{i}:\left[0, t_{f}\right] \times\left[0, x_{0}\right] \rightarrow[0, \infty)$ continuously differentiable.

The instantaneous utility function of the $i$ th player is $L^{i} ; r_{i} \geq 0$ and $S^{i}$ denote preference rate and salvage value, respectively. We impose the following hypotheses, for all $i=1, \ldots, N$, which imply risk aversion of the agents:

(H1) the function $L^{i}$ is $\mathscr{C}^{3}$ in $(0, \infty)$, monotone increasing, and strictly concave;

(H2) the function $S^{i}$ is $\mathscr{C}^{2}$ in $\left(0, x_{0}\right]$, monotone increasing, and concave.

By $\mathscr{E}^{i}, i=1, \ldots, N$, we denote the inverse of the risk aversion index of Arrow-Pratt,

$$
\mathscr{E}^{i}\left(u^{i}\right)=-L_{i}^{\prime}\left(u^{i}\right) / L_{i}^{\prime \prime}\left(u^{i}\right)
$$

With a view to analyzing this game, we consider the problem (13), (12), which in this case reads, for $i=1, \ldots, N$,

$$
\begin{aligned}
& (\partial / \partial \tau) \phi^{i}+\left(\sum_{j=1}^{N} \phi^{j}\right)(\partial / \partial x) \phi^{i}-\mathscr{E}^{i}\left(\phi^{i}\right) \sum_{j \neq i}(\partial / \partial x) \phi^{j} \\
& =r_{i} \mathscr{E}^{i}\left(\phi^{i}\right) \\
& \phi^{i}(0, x)=\varphi^{i}(x), \\
& \tau=t_{f}-t, \quad 0 \leq x \leq x_{0}, \quad \varphi^{i}(x)=\left[\left(L^{i}\right)^{\prime}\right]^{-1}\left(\left(S^{i}\right)^{\prime}(x)\right) .
\end{aligned}
$$


The following results are centered on the symmetric game:

$$
L^{i}=L, \quad S^{i}=S, \quad r_{i}=r, \quad \mathscr{U}^{i}=\mathscr{U}, \quad i=1, \ldots, N .
$$

In this case, the system (17) reduces to

$$
\begin{aligned}
& (\partial / \partial \tau) \hat{\phi}+(N \hat{\phi}-(N-1) \mathscr{E}(\hat{\phi}))(\partial / \partial x) \hat{\phi}=r \mathscr{E}(\hat{\phi}), \\
& \hat{\phi}(0, x)=\varphi(x) .
\end{aligned}
$$

Its characteristic system is given by

$$
\begin{aligned}
& \left(d / d t_{1}\right) \tau=1, \\
& \left(d / d t_{1}\right) x=N \hat{\phi}-(N-1) \mathscr{E}(\hat{\phi}), \\
& \left(d / d t_{1}\right) \hat{\phi}=r \mathscr{E}(\hat{\phi}),
\end{aligned}
$$

with initial values

$$
\begin{aligned}
& \tau(0, \beta)=0, \\
& x(0, \beta)=\beta, \\
& \hat{\phi}(0, \beta)=\varphi(\beta) .
\end{aligned}
$$

Theorem 5.1. Let us assume that the following conditions are satisfied:

(i) $\mathscr{E}^{\prime}(u) \leq N /(N-1), \forall u \in \mathscr{U}$,

(ii) $r>0 \Rightarrow \lim _{u \rightarrow+\infty} L^{\prime}(u)=0$,

(iii) $\varphi(0)=0, \mathscr{E}(0)=0$.

Then, a unique global classical solution to $(18)$ in $[0, \infty) \times\left[0, x_{0}\right]$ exists.

Proof. It is well known that there is a unique local classical solution to (18) near $\tau=0$; see Ref. 11. Our task is to extend this solution for all $\tau \geq 0$.

From (18), along the characteristic defined by

$$
(d / d \tau) x=N \hat{\phi}-(N-1) \mathscr{E}(\hat{\phi}),
$$

the solution $\hat{\phi}(\tau, x)$ satisfies

$$
(d / d \tau) \hat{\phi}=r \mathscr{E}(\hat{\phi}) .
$$

Thus, the characteristic $x=x(\tau, \beta)$ passing through any point $(0, \beta)$, $\beta \in\left[0, x_{0}\right]$, and the solution along this characteristic,

$$
\hat{\phi}(\tau, \beta)=\hat{\phi}(\tau, x(\tau, \beta)),
$$


can be determined by the Cauchy problem

$$
\begin{aligned}
& (d / d \tau) x=N \hat{\phi}-(N-1) \mathscr{E}(\hat{\phi}), \\
& (d / d \tau) \hat{\phi}=r \mathscr{E}(\hat{\phi}), \\
& \tau=0, \quad x=\beta, \quad \hat{\phi}=\varphi(\beta) .
\end{aligned}
$$

If $r=0$, then $\hat{\phi}$ is constant, and so a solution along all the time horizon exists, due to the increase in the characteristic line slopes. This fact is assured by condition (i).

On the other hand, if $r>0$, then at least locally,

$$
(1 / r) \int_{\varphi(\beta)}^{\hat{\phi}(\tau, \beta)}(1 / \mathscr{E}(z)) d z=\tau .
$$

For fixed $\beta \in\left[0, x_{0}\right]$, let us consider the following function:

$$
F_{\beta}(u)=\int_{\varphi(\beta)}^{u}(1 / \mathscr{E}(z)) d z .
$$

The function $F_{\beta}$ is $\mathscr{C}^{1}$ and monotone increasing, with $F_{\beta}(\varphi(\beta))=0$. Furthermore, assumption (ii) implies that

$$
\lim _{u \rightarrow+\infty} F_{\beta}(u)=+\infty
$$

In consequence, we can conclude that, for all $\beta \in\left[0, x_{0}\right]$, a unique $\hat{\phi}(\tau, \beta)=F_{\beta}^{-1}(\tau)$ exists for all $\tau \geq 0$. Therefore, the characteristics are

$$
x(\tau, \beta)=\beta+\int_{0}^{\tau}(N \hat{\phi}(s, \beta)-(N-1) \mathscr{E}(\hat{\phi}(s, \beta))) d s .
$$

The solution along characteristics will be well defined if and only if its envelope is empty (Ref. 12). This is accomplished if and only if

$$
\begin{aligned}
& (\partial / \partial \beta) x(\tau, \beta) \\
& =1+\int_{0}^{\tau}\left(N-(N-1) \mathscr{E}^{\prime}(\hat{\phi}(s, \beta))\right)(\partial / \partial \beta) \hat{\phi}(s, \beta) d s \\
& =1+\int_{0}^{\tau}\left(N-(N-1) \mathscr{E}^{\prime}(\hat{\phi}(s, \beta))\right)(\mathscr{E}(\hat{\phi}(s, \beta)) / \mathscr{E}(\varphi(\beta))) \varphi^{\prime}(\beta) d s \\
& \geq 0 .
\end{aligned}
$$

In fact, by (i) and taking into account that $\varphi^{\prime}(\beta) \geq 0$, we get

$$
(\partial / \partial \beta) x(\tau, \beta) \geq 1
$$


Corollary 5.1. Under the hypotheses of the last theorem, there exists a unique symmetric subgame perfect Nash equilibrium of class $\mathscr{C}^{1}$ of the game, for fixed arbitrary $t_{f}$.

Proof. In view of the fact that the Hamiltonian functions of the players are strictly concave, it is only necessary to prove the admissibility of the solution asserted by Theorem 5.1, in order to fulfill all the hypotheses in Theorem 4.1 .

Considering the proof of Theorem 5.1, it follows that, along characteristics $x(\tau, \beta)$, the solution is monotone increasing. Since the initial condition $\varphi(\beta)$ is nonnegative, it follows that the solution is nonnegative. Moreover, the unique solution along the characteristic $x(\tau, 0)$ is $\hat{\phi}(\tau, 0)=0$. This fact assures that the restriction $0 \leq x \leq x_{0}$ is fulfilled along the optimal trajectory.

Let us observe that, if the time horizon is sufficiently limited, a feasible equilibrium can still exist although

$$
\mathscr{E}(0)>0 \text { or } \varphi(0)>0 .
$$

Note that condition (i) in the above theorem is a similar condition to that required for two players in Ref. 10, because

$$
\mathscr{E}^{\prime}(u) \leq N /(N-1) \Leftrightarrow E^{\prime}(u) \geq[E(u) / u][1-E(u) N /(N-1)],
$$

where

$$
E(u)=u / \mathscr{E}(u)
$$

is the elasticity of the marginal utility. If the relative risk aversion coefficient is decreasing [that is, $E^{\prime}(u) \leq 0$ ], then (22) implies that

$$
E(u) \geq 1-1 / N \text {. }
$$

When $N=1$, that is, in the optimal control problem, there is no restriction on $\mathscr{E}$ related to the existence of $\hat{\phi}$. In the game framework, if the slope of $\mathscr{E}$ is greater than $N /(N-1)$, then the solution becomes discontinuous in finite time. In fact, it is the gradient of the strategy which becomes unbounded. This behavior is a consequence of the extensive exploitation of the resource under noncooperative management. The speed of the change in each player's strategy increases without bound. This fact is due to the aim of adapting his or her own strategy to the competitors' strategies in an optimum way.

We can provide implicit expressions for the solution in some important cases. 
Theorem 5.2. The unique global classical solution of (18) is determined as follows:

(i) constant elasticity of the marginal utility,

$$
\begin{aligned}
& E(u)=-\gamma, \quad \gamma \in[-1,-1+1 / N], \quad r>0, \\
& \hat{\phi}(\tau, x)=\exp (-(r / \gamma) \tau) \varphi[x+(\gamma / r)(1-\exp ((r / \gamma) \tau)) \\
& \times((N-1) / \gamma+N) \hat{\phi}(\tau, x)]
\end{aligned}
$$

(ii) elasticity of the marginal utility satisfying (22) and $r=0$,

$$
\hat{\phi}(\tau, x)=\varphi[x+\tau((N-1) \mathscr{E}(\hat{\phi}(\tau, x))-N \hat{\phi}(\tau, x))] .
$$

Furthermore, in all cases the solution is monotone increasing with respect to $x$. In (i), if the solution is $\mathscr{C}^{2}$ and $\varphi^{\prime \prime} \geq 0$, then it is convex with respect to $x$. In (ii), if the solution is $\mathscr{C}^{2}, \varphi^{\prime \prime} \geq 0$, and $\mathscr{E}^{\prime \prime} \geq 0$, then it is convex with respect to $x$.

Proof. It is easy to show that, in all cases, the hypotheses of Theorem 5.1 are fulfilled. The respective expressions are obtained after integration in the ordinary differential equations system (19)-(20). The second part of the theorem is straightforward by differentiation of (23b)-(24).

Next, we study the properties of the equilibrium along the optimal trajectory. We define $\phi^{*}(t)=\hat{\phi}\left(t, x^{*}(t)\right)$, where $x^{*}$ is the unique solution to the closed-loop equation

$$
\dot{x}(t)=-N \hat{\phi}(t, x(t)) .
$$

Theorem 5.3. In all cases considered in Theorem 5.2, $x^{*}$ is a convex function.

Proof. Clearly, it is sufficient to prove that $\phi^{*}$ is monotone decreasing. The relation

$$
\dot{\phi}^{*}(t)=(\partial / \partial t) \hat{\phi}\left(t, x^{*}(t)\right)-N \hat{\phi}\left(t, x^{*}(t)\right)(\partial / \partial x) \hat{\phi}\left(t, x^{*}(t)\right) \leq 0
$$

shows this fact.

Other important aspects of resource games are the effects on competitive extraction of variations in the number of players, utility function, and salvage value. We carry out an analytical study in the following theorems. 
Theorem 5.4. Consider the following Cauchy problems:

$$
\text { (P1) } \begin{array}{ll} 
& (\partial / \partial \tau) u+f(u)(\partial / \partial x) u=h(u), \\
& u(0, x)=\varphi(x) \\
\text { (P2) } \quad & (\partial / \partial \tau) v+g(v)(\partial / \partial x) v=h(v), \\
& v(0, x)=\psi(x) .
\end{array}
$$

Here, $\tau \geq 0, x \in \mathbb{R} ; f, g, \varphi, \psi$ are $\mathscr{C}^{l}$ functions, monotone increasing, and satisfying $f \geq g$ and $\varphi \leq \psi$. Furthermore, let $h$ be a nonnegative continuous function. If $u, v$ are the unique global classical solutions to (P1) and (P2) respectively, then $u \leq v$.

Proof. Suppose that there exist $\tau>0$ and $x \in \mathbb{R}$ such that $u(\tau, x)>v(\tau, x)$. Let $\beta$ and $\beta^{\prime}$ be real numbers such that the characteristics $x(s, \beta)$ and $\hat{x}\left(s, \beta^{\prime}\right)$, associated to (P1) and (P2) passing through $(0, \beta)$ and $\left(0, \beta^{\prime}\right)$, respectively, intersect in $(\tau, x)$. Then, note that

$$
\begin{aligned}
& x(\tau, \beta)=\beta+\int_{0}^{\tau} f(u(s, x(s, \beta))) d s, \\
& \hat{x}\left(\tau, \beta^{\prime}\right)=\beta^{\prime}+\int_{0}^{\tau} g\left(v\left(s, \hat{x}\left(s, \beta^{\prime}\right)\right)\right) d s,
\end{aligned}
$$

and by subtraction,

$$
\beta-\beta^{\prime}=\int_{0}^{t}\left[g\left(v\left(s, \hat{x}\left(s, \beta^{\prime}\right)\right)\right)-f(u(s, x(s, \beta)))\right] d s .
$$

Now, taking into account that $\varphi \leq \psi$ and that these functions are increasing, then it follows that $\beta>\beta^{\prime}$, and due to the nonnegativity of $h$,

$$
u(s, x(s, \beta))>v\left(s, \hat{x}\left(s, \beta^{\prime}\right)\right), \quad \forall s \in[0, \tau] .
$$

So, the right-hand side of (25) is positive, in contradiction with the hypotheses.

Theorem 5.5. Consider problems (P1) and (P2) with $h \equiv 0$. Suppose that $\tau \geq 0, x \in \mathbb{R}$, and that $f, g, \varphi, \psi$ are $\mathscr{C}^{1}$ functions, monotone increasing, with $\varphi, \psi$ bounded, and satisfying $\max \varphi \leq \min \psi$. If $u, v$ are the unique global classical solutions to (P1) and (P2) respectively, then $u \leq v$.

Proof. The conditions imposed assure existence and uniqueness of solution for both equations (Ref. 12). Furthermore, the solution is constant 
along characteristics that are straight lines. The values of the solution throughout the characteristic passing $(0, \beta)$ are $\varphi(\beta)$ and $\psi(\beta)$, respectively. In consequence,

$$
u(\tau, x) \leq \max \varphi \leq \min \psi \leq v(\tau, x), \quad \text { for all } \tau \geq 0, x \in \mathbb{R} .
$$

Corollary 5.2. Let us suppose that condition (22) holds. Then, the depletion rate of each player's resource increases (decreases) when $N$ grows, if and only if $E<1(E>1)$. When $E=1$, the extraction is independent of the player number.

Proof. Let us define

$$
\alpha_{N}(u)=N u-(N-1) \mathscr{E}(u), \quad N>1 .
$$

We assume that $N>M$. Then, one can get

$$
\alpha_{N}(u)\left\{\begin{array}{l}
< \\
= \\
>
\end{array}\right\} \alpha_{M}(u) \Leftrightarrow E\left\{\begin{array}{l}
< \\
= \\
>
\end{array}\right\} 1 .
$$

We apply Theorem 5.4, with

$$
f=\alpha_{N}, \quad g=\alpha_{M},
$$

to reach the desired conclusion.

The results established show that, for $E<1$, the entry of new players would speed the extraction of all the players' resources, whereas, if $E=1$, the entry of new players would not change the resource depletion rate.

Now, we proceed to compare two separate models of resource extraction characterized by functions $E^{(1)}, E^{(2)}$ and final conditions $\varphi^{(1)}, \varphi^{(2)}$, with the same number of players.

Corollary 5.3. Suppose that (22) hold. Then:

(i) $\varphi^{(1)} \equiv \varphi^{(2)}$ and $E^{(1)} \geq E^{(2)}$, with $E^{(1)}(u)>E^{(2)}(u)$ for some $u$, imply $\hat{\phi}^{(1)} \leq \hat{\phi}^{(2)}$, with $\hat{\phi}^{(1)}(t, x)<\hat{\phi}^{(2)}(t, x)$ for some $(t, x)$.

(ii) $E^{(1)} \equiv E^{(2)}$ and $\varphi^{(1)} \leq \varphi^{(2)}$, with $\varphi^{(1)}<\varphi^{(2)}$ for some $x$, imply $\hat{\phi}^{(1)} \leq \hat{\phi}^{(2)}$, with $\hat{\phi}^{(1)}(t, x)<\hat{\phi}^{(2)}(t, x)$ for some $(t, x)$. 5.4 .

Proof. The proof follows the lines of the demonstration of Theorem 
The first result establishes that for identical boundary functions, the greater the marginal utility elasticity, the smaller the exploitation activity rate. The second one shows a more rapid extraction speed for a bigger boundary function when the elasticity of the marginal utility remains the same.

Remark 5.1. If we consider the symmetric cooperative game, then we have the associated Cauchy problem

$$
\begin{aligned}
& (\partial v / \partial \tau)+N v(\partial v / \partial x)=r \mathscr{E}(v), \\
& v(0, x)=\left(L^{\prime}\right)^{-1}\left(N S^{\prime}(x)\right) .
\end{aligned}
$$

Now, using Theorem 5.4, we obtain that the resource extraction is smaller than in the noncooperative game. The smaller exploitation intensity in the cooperative case allows the possibility of leaving more resources to be extracted in the future.

Remark 5.2. The renewable resource game with linear recruit function and isoelastic utility function,

$$
\begin{array}{ll}
\max _{u^{i}} & \int_{0}^{t_{f}} \exp \left(-r_{i} t\right)\left(u^{i}\right)^{1+\gamma_{1}} d t+S^{i}\left(t_{f}, x\left(t_{f}\right)\right), \quad i=1, \ldots, N, \\
\text { s.t. } & \dot{x}=a(\bar{x}-x)-\sum_{i=1}^{N} u^{i}, \\
& x(0)=x_{0}>0, \quad \gamma_{i}>0,
\end{array}
$$

can easily be converted to an autonomous nonrenewable resource model, such as the one studied in this section, by means of a one-to-one state and control transformation. Let

$$
y=(x-\bar{x}) \exp (a t), \quad v^{i}=\exp (a t) u^{i}, i=1, \ldots, N .
$$

Then, the game becomes

$$
\begin{array}{ll}
\max _{u^{i}} & \int_{0}^{t_{f}} \exp \left(-r_{i}^{\prime} t\right)\left(v^{i}\right)^{1+\gamma_{i}} d t+S^{i}\left(t_{f}, y\left(t_{f}\right)\right), \quad i=1, \ldots, N, \\
\text { s.t. } & \dot{y}=-\sum_{i=1}^{N} v^{i}, \\
& y(0)=x_{0}-\bar{x}, \\
& \hat{S}^{i}(t, y)=S^{i}(t, x)=S^{i}(t, y \exp (-a t)+\bar{x}), \quad r_{i}^{\prime}=r_{i}+a\left(1+\gamma_{i}\right) .
\end{array}
$$


In consequence, all the results displayed in this section for the symmetric game are applicable to this model. In addition, the optimal strategy for each player is

$$
u(t, x)=\exp (-a t) v(t,(x-\bar{x}) \exp (a t)) .
$$

\section{Conclusions}

We have given necessary and sufficient conditions to characterize subgame perfect Nash equilibria. This way allows us to find the equilibria explicitly in some games where classical methods, such as the HamiltonJacobi-Bellman equation, are usually more complex to apply. Moreover, if the explicit solution cannot be computed, our method helps to analyze the qualitative features of the equilibrium.

This approach for the determination of the Nash equilibrium in feedback strategies does not need the usual concavity hypotheses on the player's Hamiltonians.

\section{References}

1. Friedman, A., Differential Games, Wiley, New York, New York, 1971.

2. Leitmann, G., Cooperative and Noncooperative Many-Player Differential Games, Springer Verlag, New York, New York, 1974.

3. Melmmann, A., Applied Differential Games, Plenum Press, New York, New York, 1988.

4. Bourdache-Siguerdidjane, H., On Applications of a New Method for Computing Optimal Nonlinear Feedback Controls, Optimal Control Applications and Methods, Vol. 8, pp. 397-409, 1987.

5. Bourdache-Siguerdidjane, H., and Fliess, M., Optimal Feedback Control of Nonlinear Systems, Automatica, Vol. 23, pp. 365-372, 1987.

6. Courant, R., and Hilbert, D., Methods of Mathematical Physics, Vol. 2, Wiley, New York, New York, 1962.

7. Willemstein, A. P., Optimal Regulation of Nonlinear Dynamical Systems on a Finite Interval, SIAM Journal on Control and Optimization, Vol. 15, pp. 1050 1069, 1977.

8. BAÇAR, T., and OlsDer, G. J., Dynamic Noncooperative Game Theory, 2nd Edition, Academic Press, London, England, 1995.

9. Fershtman, C., Identification of Classes of Differential Games for Which the Open Loop Is a Degenerate Feedback Nash Equilibrium, Journal of Optimization Theory and Applications, Vol. 55, pp. 217-231, 1987. 
10. Clemhout, S., and WAN, H. Y., Differential Games-Economic Applications, Handbook of Game Theory, Edited by R. J. Aumann and S. Hart, North Holland, Amsterdam, Holland, Vol. 2, pp. 801-825, 1994.

11. JoHN, F., Partial Differential Equations, Springer Verlag, New York, New York, 1971.

12. LI, T. T., Global Classical Solutions for Quasilinear Hyperbolic Systems, Wiley, New York, New York, 1994. 